\title{
碲纳米线柔性薄膜的制备及其热电性能
}

\author{
徐海丰, 侯成义, 张青红, 李耀刚, 王宏志 \\ (东华大学 材料科学与工程学院, 纤维材料改性国家重点实验室, 上海 201620)
}

摘 要: 柔性热电器件能够直接将人体热量转化为电能, 因而受到广泛的关注。本研究采用水热法合成了碲纳米线, 探究了水热温度和反应溶液的还原性强弱(添加抗坏血酸与否)对碲纳米线形貌及热电性能的影响。与强还原性反应 液(添加抗坏血酸)中制备的碲纳米线相比，弱还原性反应液(未添加抗坏血酸)中制备的碲纳米线具有较高的长径比， 最高可达 200 , 其组装的薄膜具有更高的电导率, 达到 $26 \mathrm{~S} \cdot \mathrm{m}^{-1}$ 。进一步研究了成膜工艺对碲纳米线薄膜热电性能 的影响, 发现湿压法可提升薄膜的微观致密度, 使薄膜中碲纳米线之间的微观连接更为紧密, 从而改善了薄膜的 载流子迁移率和载流子浓度, 使薄膜的电导率提升了 18.3 倍, 达到 $476 \mathrm{~S} \cdot \mathrm{m}^{-1}$, 塞贝克系数为 $282.9 \mu \mathrm{V} \cdot \mathrm{K}^{-1}$, 功率因 子达到 $38 \mu \mathrm{W} \cdot \mathrm{m}^{-1} \cdot \mathrm{K}^{-2}$ 。

关 键 词: 柔性热电; 湿压; 碲纳米线

中图分类号: TQ174 文献标识码: A

\section{Preparation and Thermoelectric Performance of Tellurium Nanowires-based Thin-Film Materials}

\section{XU Haifeng, HOU Chengyi, ZHANG Qinghong, LI Yaogang, WANG Hongzhi}

(State Key Laboratory for Modification of Chemical Fibers and Polymer Materials, College of Materials Science and Engineering, Donghua University, Shanghai 201620, China)

\begin{abstract}
Flexible thermoelectric devices have received a great deal of interest due to their capability of direct convert human body heat into electrical energy. In this work, we synthesized the tellurium nanowires by using hydrothermal method. The effects of hydrothermal temperature and reducibility of the reaction solution (with or without ascorbic acid) on morphology and thermoelectric properties of tellurium nanowires were investigated. Compared to the nanowires prepared in strong reducing solution, those prepared in relatively weak reducing solution (without ascorbic acid) reveal a higher aspect ratio up to 200 and the as-assembled film exhibits better electrical conductivity up to $26 \mathrm{~S} \cdot \mathrm{m}^{-1}$. It is also found that the wet-press method can enhance the micro-densification of the film, resulting in a tighter connection among the micro-nanowires. Therefore, the carrier mobility and carrier concentration of the tellurium nanowire film are significantly increased. As a result, its electrical conductivity is improved by 18.3 times, reached $476 \mathrm{~S} \cdot \mathrm{m}^{-1}$. Simultaneously the optimal tellurium nanowire film exhibits good performances including Seebeck coefficient $\left(282.9 \mu \mathrm{V} \cdot \mathrm{K}^{-1}\right)$ and power factor $\left(38 \mu \mathrm{W} \cdot \mathrm{m}^{-1} \cdot \mathrm{K}^{-2}\right)$.
\end{abstract}

Key words: flexible thermoelectric; wet-press; tellurium nanowire

收稿日期：2019-10-28; 收到修改稿日期：2020-01-09

基金项目: 国家自然科学基金(51603037, 51873033) National Natural Science Foundation of China $(51603037,51873033)$

作者简介：徐海丰(1993-), 男, 硕士研究生. E-mail: 15221813638@163.com XU Haifeng (1993-), male, Master candidate. E-mail: 15221813638@163.com

通讯作者: 侯成义, 副研究员. E-mail: hcy@dhu.edu.cn; 李耀刚, 教授. E-mail: yaogang_li@dhu.edu.cn HOU Chengyi, associate professor. E-mail: hcy@dhu.edu.cn; LI Yaogang, professor. E-mail: yaogang_li@dhu.edu.cn 
可穿戴电子设备的蓬勃发展刺激了市场对持续 供能设备的需求, 特别对于户外演练、体征监测等 全天候工作的可穿戴电子设备, 频繁地更换电池或 充电限制了它们在军事领域和医疗领域的广泛应 用 ${ }^{[1-2]}$ 。因此, 迫切需要发展可持续供能并兼具柔性 的可穿戴能源材料与器件 ${ }^{[3-4]}$ 。人体作为一种持续的 热源, 即使处于静止状态, 其辐射热损失也占到 $40 \% \sim 60 \%$, 有潜力成为智能手表、智能服装、智能 输药系统等可穿戴电子设备的理想能量来源 ${ }^{[5]}$ 。热 电材料作为一种极具发展前景的可穿戴能量采集 材料, 可通过人体皮肤与周围环境的温度梯度实 现可穿戴电子器件的自供电, 因而受到越来越多 的关注 ${ }^{[6-7]}$ 。

无机块体热电材料, 如 $\mathrm{Sb}_{2} \mathrm{Te}_{3} 、 \mathrm{Bi}_{2} \mathrm{Te}_{3}$ 等 ${ }^{[8]}$ 具有 较高的热电性能, 但缺乏柔性, 很难应用到柔性可 穿戴领域 ${ }^{[9]}$ 。有机热电材料如聚苯胺(PANI)、聚 3, 4乙烯二氧噻吩/聚苯乙烯磺酸盐(PEDOT:PSS)等具 有柔性和轻质的特性 ${ }^{[10-11]}$, 满足柔性热电材料的需 求, 然而功率因子大多相对较低 $\left(\sim 4.5 \mu \mathrm{W} \cdot \mathrm{m}^{-1} \cdot \mathrm{K}^{-2}\right)^{[12]}$, 制约了其在柔性热电领域的应用。因此, 研究者通 过低维化和复合的方法来制备性能优异的柔性热电 材料 ${ }^{[13-14]}$ 。Gao 等 ${ }^{[15]}$ 利用碲纳米线复合还原氧化石 墨烯制备柔性薄膜, 功率因子达到 $80 \mu \mathrm{W} \cdot \mathrm{m}^{-1} \cdot \mathrm{K}^{-2}$ 。 Song 等 ${ }^{[16]}$ 利用碲纳米棒复合 PEDOT:PSS 制备柔性 热电薄膜, 功率因子达到 $50 \mu \mathrm{W} \cdot \mathrm{m}^{-1} \cdot \mathrm{K}^{-2}$ 。目前碲纳 米材料与碳材料或有机热电材料复合能够制备性能 优异的柔性热电材料, 但是碲纳米材料本身的热电 性能还有待进一步提高。See 等 ${ }^{[17]}$ 采用水热法制备 了碲纳米线, 测试了碲纳米线薄膜的热电性能, 其 电导率为 $8 \mathrm{~S} \cdot \mathrm{m}^{-1}$, 塞贝克系数为 $408 \mu \mathrm{V} \cdot \mathrm{K}^{-1}$, 功率 因子为 $2.7 \mu \mathrm{W} \cdot \mathrm{m}^{-1} \cdot \mathrm{K}^{-2}$ 。 $\mathrm{Gao}$ 等 ${ }^{[18]}$ 利用高温液相法 制备了碲纳米线, 其薄膜电导率为 $4 \mathrm{~S} \cdot \mathrm{m}^{-1}$, 塞贝克 系数为 $359 \mu \mathrm{V} \cdot \mathrm{K}^{-1}$, 功率因子为 $0.53 \mu \mathrm{W} \cdot \mathrm{m}^{-1} \cdot \mathrm{K}^{-2}$ 。 碲纳米线薄膜的电导率较低, 限制了其在柔性热电 领域的应用。

本研究提出利用水热法制备碲纳米线, 通过调 控水热溶液的还原性强弱, 制备长径比较高的碲纳 米线, 并采用简单的湿压处理方法对碲纳米线加工 成膜, 以提高碲纳米线薄膜的电导率, 从而获得兼 具柔性和高电导率的碲纳米线薄膜。

\section{1 实验方法}

\section{1 实验原料}

氧化碲、氢氧化钾和聚乙烯吡咯烷酮 $(M \mathrm{w}=$ 40000)购自阿拉丁公司。抗坏血酸、乙二醇和无水
乙醇购自国药集团化学试剂有限公司。

\section{2 碲纳米线的制备及其柔性薄膜的组装}

碲纳米线的制备：将 $0.1675 \mathrm{~g}$ 氧化碲、 $0.1671 \mathrm{~g}$ 氢氧化钾加入 $30 \mathrm{~mL}$ 乙二醇溶剂中, 加热并摚拌, $85{ }^{\circ} \mathrm{C}$ 时得到透明溶液。此时加入 $0.1 \mathrm{~g}$ 聚乙烯吡咯 烷酮(PVP), 并使其完全溶解。最后将溶液转移至 $100 \mathrm{~mL}$ 的聚四氟乙烯水热釜中并在烘箱中加热 $24 \mathrm{~h}$ ，水热温度分别为 $140 、 160 、 180 、 200{ }^{\circ} \mathrm{C}$ 。反 应结束后用超纯水离心、清洗 3 次以上, 最后将洗 净的碲纳米线分散至无水乙醇中并在冰箱中冷冻保 存。水热反应液的还原性可通过额外加入还原剂进 行调控, 本工作对照组采取的实验方案为: 在上述 水热反应液中加入 $1.5 \mathrm{~mL} 20 \mathrm{wt} \%$ 抗坏血酸溶液。

根据水热反应溶液的组成, 将未添加抗坏血酸 的反应溶液在 $160{ }^{\circ} \mathrm{C}$ 下反应 $24 \mathrm{~h}$ 制备的碲纳米线 命名为 $\mathrm{TN} 160$, 添加抗坏血酸溶液在 $160{ }^{\circ} \mathrm{C}$ 下反应 $24 \mathrm{~h}$ 制备的碲纳米线命名为 ATN160。

碲纳米线薄膜的制备：首先将合成的碲纳米线 分散在超纯水中并水浴超声 $0.5 \mathrm{~h}$, 然后将所得的碲 纳米线分散液真空抽滤 $2 \mathrm{~h}$, 将真空抽滤制成的半 干薄膜在压片机下以不同的压力 $(10 、 20 、 30 \mathrm{MPa})$ 处理 $10 \mathrm{~min}$, 最后在 $120{ }^{\circ} \mathrm{C}$ 的真空烘箱中处理 $3 \mathrm{~h}$ 得到碲纳米线薄膜(图 1)。

\section{3 表征与测试}

通过日本 Hitachi 公司的 Model S-4800 型场发 射扫描电子显微镜对样品的形貌、薄膜的表面和断 面进行表征。通过赛默飞公司的 Talos F200S 场发射 透射电子显微镜对样品进行形貌的高分辨表征。通 过日本 Rigaku 的 $28 \mathrm{D} / \mathrm{max}-2550 \mathrm{~V}$ 型 X 射线衍射仪 对样品的晶体结构进行分析, 测试条件为管压 $40 \mathrm{kV}$, 电流 $300 \mathrm{~mA}, \mathrm{Cu} \mathrm{K \alpha}, \lambda=0.154056 \mathrm{~nm}$, 扫描速度为 $0.02\left({ }^{\circ}\right) / \mathrm{s}$, 扫描范围为 $10^{\circ} \sim 60^{\circ}$ 。通过四探针仪器 (MCP-T360)测量碲纳米线薄膜的电阻率, 并换算成 电导率。基于实验室的前期工作 ${ }^{[19]}$, 用自主搭建的

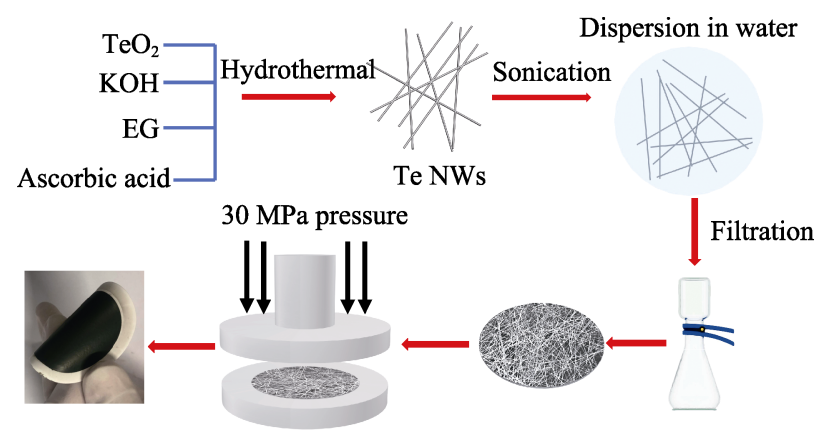

图 1 碲纳米线的制备及其柔性薄膜组装流程示意图

Fig. 1 Schematic illustration for the preparation of the Te nanowire and flexible film 
测试系统测试碲纳米线薄膜的塞贝克系数。具体为: 采用 Peltier 冷却器和加热器控制样品两端的温度, 用 KEITHLEY 2700 (A Tektronix)数据采集系统测量 薄膜两端的开路电压, 同时用热电偶测量薄膜两端 的实时温度, 计算后获得塞贝克系数。采用 Lake Shore (7704)霍尔效应测试系统测试薄膜的载流子 浓度和载流子迁移率。在薄膜两端涂上银浆, 采用 万用表 $\left(\mathrm{VC} 890 \mathrm{C}^{+}\right)$测量薄膜电阻。使用分析天平 (AL204)测试薄膜的质量。

\section{2 结果与讨论}

\section{1 碲纳米线的物相与形貌表征}

图 2(a d)分别为未添加抗坏血酸的反应溶液在 140、160、180、 $200{ }^{\circ} \mathrm{C}$ 反应 $24 \mathrm{~h}$ 制备的碲纳米线 的 FESEM 照片, 图 2(e h) 分别为添加抗坏血酸的反 应溶液在 140、160、180、200 ${ }^{\circ} \mathrm{C}$ 反应 $24 \mathrm{~h}$ 制备的 碲纳米线的 FESEM 照片。

通过氧化碲、抗坏血酸、乙二醇、氢氧化钠、 PVP 制备碲纳米线, 其化学反应式如下:

$$
\begin{gathered}
\mathrm{TeO}_{2}+2 \mathrm{OH}^{-} \rightarrow \mathrm{TeO}_{3}{ }^{2-}+\mathrm{H}_{2} \mathrm{O} \\
\mathrm{TeO}_{3}{ }^{2-}+\mathrm{C}_{6} \mathrm{H}_{8} \mathrm{O}_{6} \rightarrow \mathrm{Te}+\mathrm{C}_{6} \mathrm{H}_{4} \mathrm{O}_{6}+2 \mathrm{OH}^{-}+\mathrm{H}_{2} \mathrm{O}
\end{gathered}
$$

当氢氧化钾和氧化碲被加入到乙二醇中后, 溶 液中产生大量的 $\mathrm{TeO}_{3}{ }^{2-}$, 还原剂抗坏血酸将 $\mathrm{TeO}_{3}{ }^{2-}$ 还原成碲原子, 而当反应溶液中未添加还原剂抗坏 血酸时, 弱还原性的乙二醇充当了还原剂的角色, 同样可以将 $\mathrm{TeO}_{3}{ }^{2-}$ 还原成碲原子, 但是其还原速度 较慢, 溶液中碲的饱和度较低, 此时晶体生长优于 晶体成核 ${ }^{[20]}$, 在结构导向剂 PVP 的作用下缓慢生长
成长径比较高的碲纳米线, 如图 $2(\mathrm{a} \sim \mathrm{d})$ 所示, 其 中 $160{ }^{\circ} \mathrm{C}$ 下制备的碲纳米线长径比最高, 约为 200 (图 2(b))。当溶液中添加还原剂抗坏血酸时, 由于其 还原能力较强, 从而加速了 $\mathrm{TeO}_{3}{ }^{2-}$ 的还原, 使碲的 饱和度过高, 此时晶核的生成量过多, 易得到长度 较短的碲纳米线 ${ }^{[21]}$, 长径比大多较低, 如图 $2(\mathrm{e} \sim \mathrm{h})$ 所示, 其中 $160{ }^{\circ} \mathrm{C}$ 下制备的碲纳米线长径比约为 40 (图 2(f))。

图 3 为 TN160 的 XRD 图谱, 所有的衍射峰都 与碲的三方相对应, 衍射峰尖锐, 表明碲纳米线结 晶性良好。

TN160 的 TEM 表征结果如图 4 所示, 碲纳米线 的长度约为 $10 \mu \mathrm{m}$ 。图 4(b)中相邻晶格条纹之间的 间距为 $0.59 \mathrm{~nm}$, 对应碲纳米线的 $(001)$ 晶面, 说明 碲纳米线沿[001]晶向生长 ${ }^{[22]}$ 。图 4(c) 为高倍 TEM 照片的傅里叶变换图, 点阵结构规则, 说明碲纳米 线为单晶。

\section{2 碲纳米线薄膜的热电性能分析}

为了确定合成条件对碲纳米线热电性能的影响, 对不同碲纳米线组装的薄膜进行热电性能测试, 结 果如图 5 所示。从图 5(a)中可以看出, 碲纳米线薄 膜的塞贝克系数与反应温度、还原剂抗坏血酸相关 性不大。图 5(b)是不同反应条件下制备的碲纳米线 所组装的薄膜的电导率, 可以看出还原剂抗坏血酸 对薄膜的电导率影响较大, 反应溶液中未添加还 原剂所制备的薄膜具有较高的电导率。对比不同 温度下制备的碲纳米薄膜, 可以看出在 $160{ }^{\circ} \mathrm{C}$ 未添 加抗坏血酸制备的薄膜具有最高的电导率, 达到 $26 \mathrm{~S} \cdot \mathrm{m}^{-1}$ 。不同条件下制备的碲纳米线组装的薄膜的
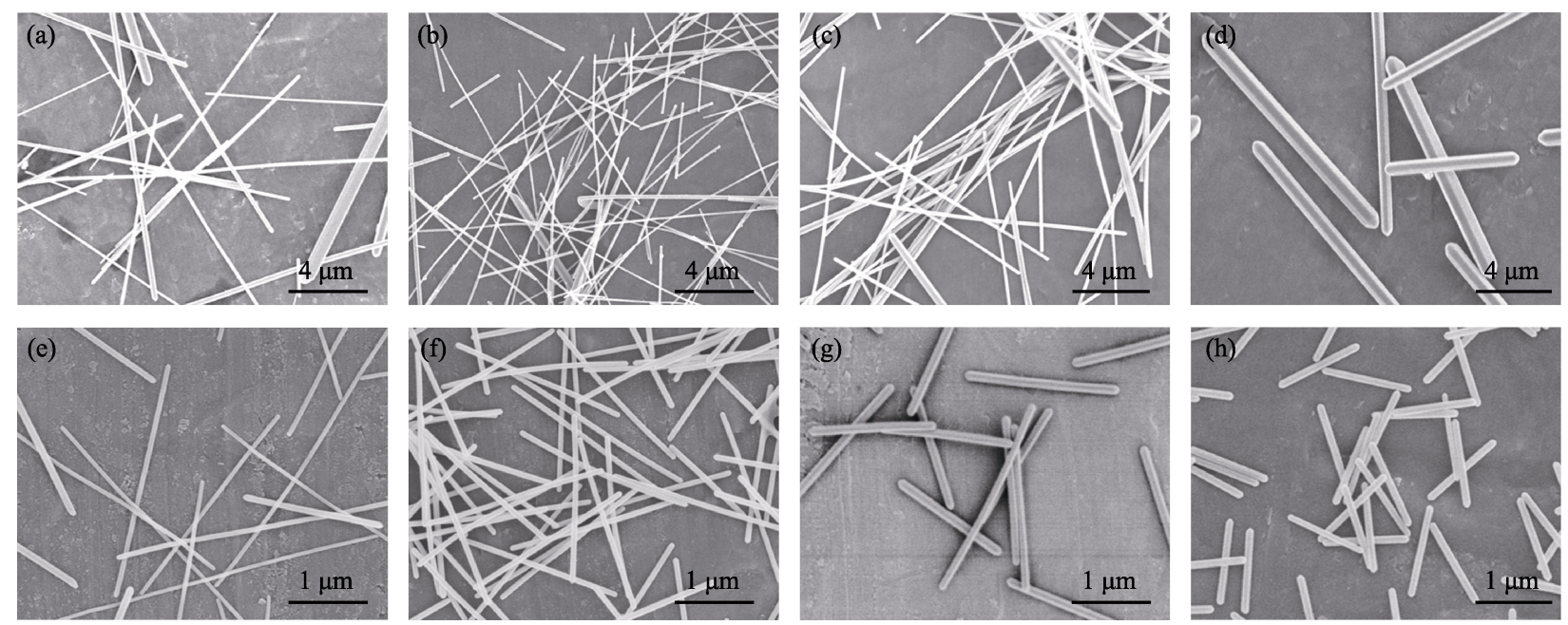

图 2 未添加抗坏血酸的反应溶液在(a) 140、(b) 160、(c) 180、(d) $200{ }^{\circ} \mathrm{C}$ 制备的碲纳米线的 FESEM 照片; 添加抗坏血酸的反应溶液在(e) 140、(f) 160、(g) 180、(h) $200{ }^{\circ} \mathrm{C}$ 制备的碲纳米线的 FESEM 照片

Fig. 2 FESEM images of Te nanowires obtained by the solution without ascorbic acid at (a) 140 , (b) 160 , (c) 180 and (d) $200{ }^{\circ} \mathrm{C}$, and with ascorbic acid at (e) 140 , (f) 160 , (g) 180 and (h) $200{ }^{\circ} \mathrm{C}$ 


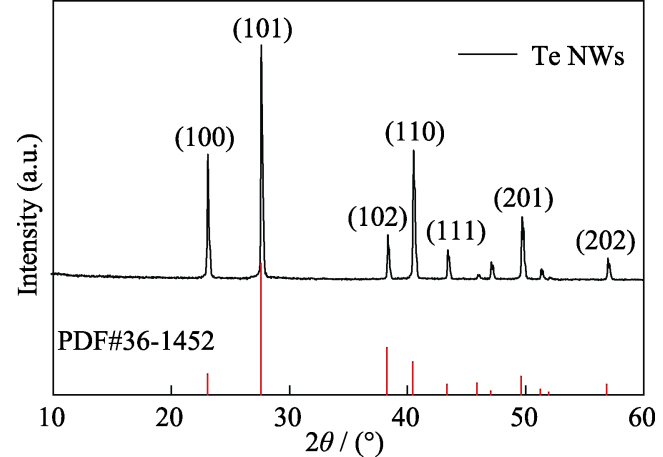

图 3 TN160 薄膜的 XRD 图谱

Fig. 3 XRD pattern of TN160 film

功率因子如图 5(c)所示, TN160 薄膜具有较高的功 率因子, 热电性能最优。为了探究 TN160 薄膜具有
较高电导率的原因，分别对 ATN160、TN160 薄膜进 行 FESEM 表征(图 5(d,g)) 和电阻测试(图 5(e, f))。 TN160 薄膜中碲纳米线之间的连接较为紧密, 且纳 米线更长，这些特征都有利于载流子传输。在 TN160 薄膜表面测得的电阻显著低于 ATN160 薄膜, 与电导率测试的结论相符。

为了进一步增加薄膜的微观致密度以提高电导 率，采用湿压的方式处理真空抽滤法制备的 TN160 薄膜。未湿压处理的薄膜电阻约为 $1.21 \mathrm{k} \Omega$ (图 6(a)), 薄膜内部的纳米线连接较为松散(图 6(e))。与未湿压 处理的薄膜相比, $10 \mathrm{MPa}$ 湿压处理的薄膜电阻 $(0.53 \mathrm{k} \Omega$ ) 明显降低(图 6(b)), 主要得益于湿压处理后 薄膜内部纳米线之间连接较为紧密(图 6(f))。20 MPa
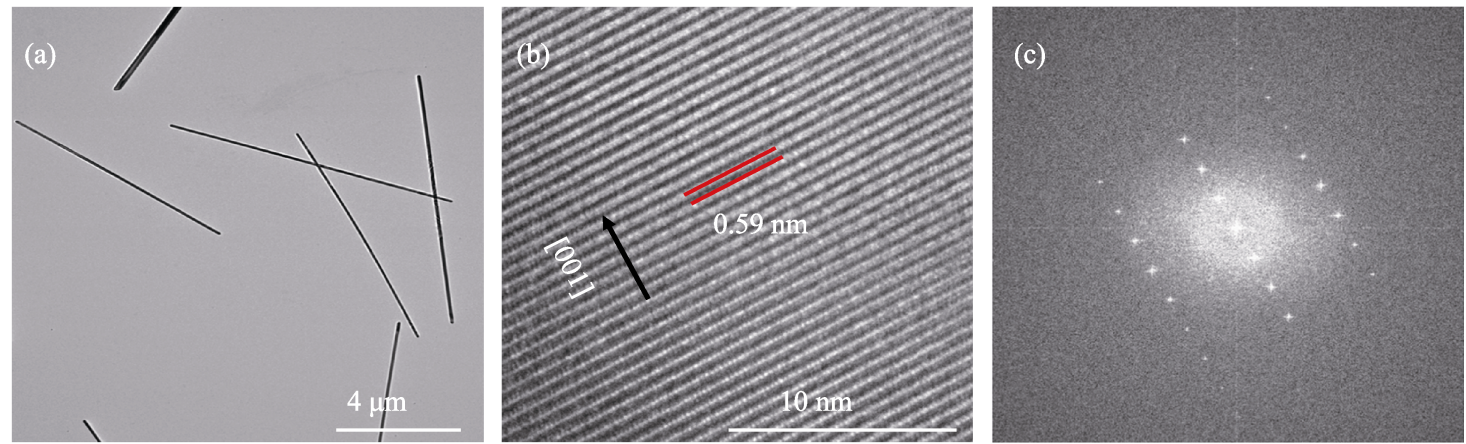

图 4 TN160 的(a)低倍、(b)高倍 TEM 照片和(c)傅里叶变换图

Fig. 4 (a) Low-magnification, (b) high-magnification TEM images and (c) Fourier transformation pattern of TN160
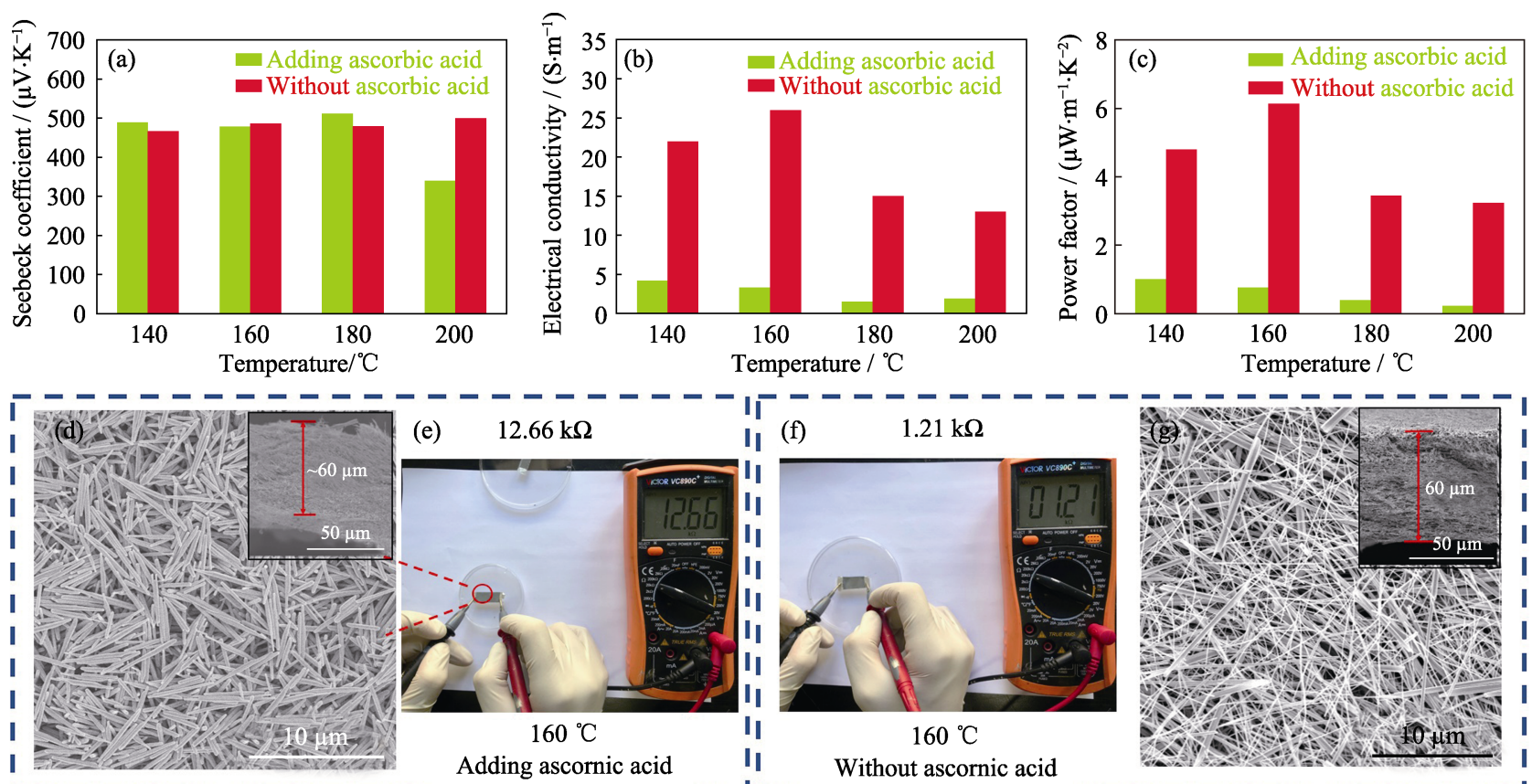

Adding ascornic acid

Without ascornic acid

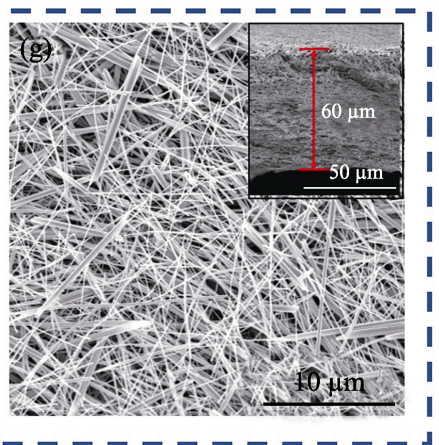

图 5 不同条件下制备的碲纳米线薄膜的(a)塞贝克系数、(b)电导率和(c)功率因子; (d) ATN160 和 $(\mathrm{g})$ TN160 薄膜的表面 FESEM 照片, 插图为对应薄膜的断面 FESEM 照片; (e) ATN160 和(f)TN160 薄膜 $(1 \mathrm{~cm} \times 2 \mathrm{~cm})$ 的电阻测试的数码照片

Fig. 5 (a) Seebeck coefficients, (b) electrical conductivities, (c) power factors of Te nanowire films prepared under different conditions; Surface FESEM images of (d) ATN160 and (g) TN160 films with insets showing corresponding cross-sectional FESEM images; Digital photos of film $(1 \mathrm{~cm} \times 2 \mathrm{~cm})$ resistance tests for (e) ATN160 and (f) TN160 films 

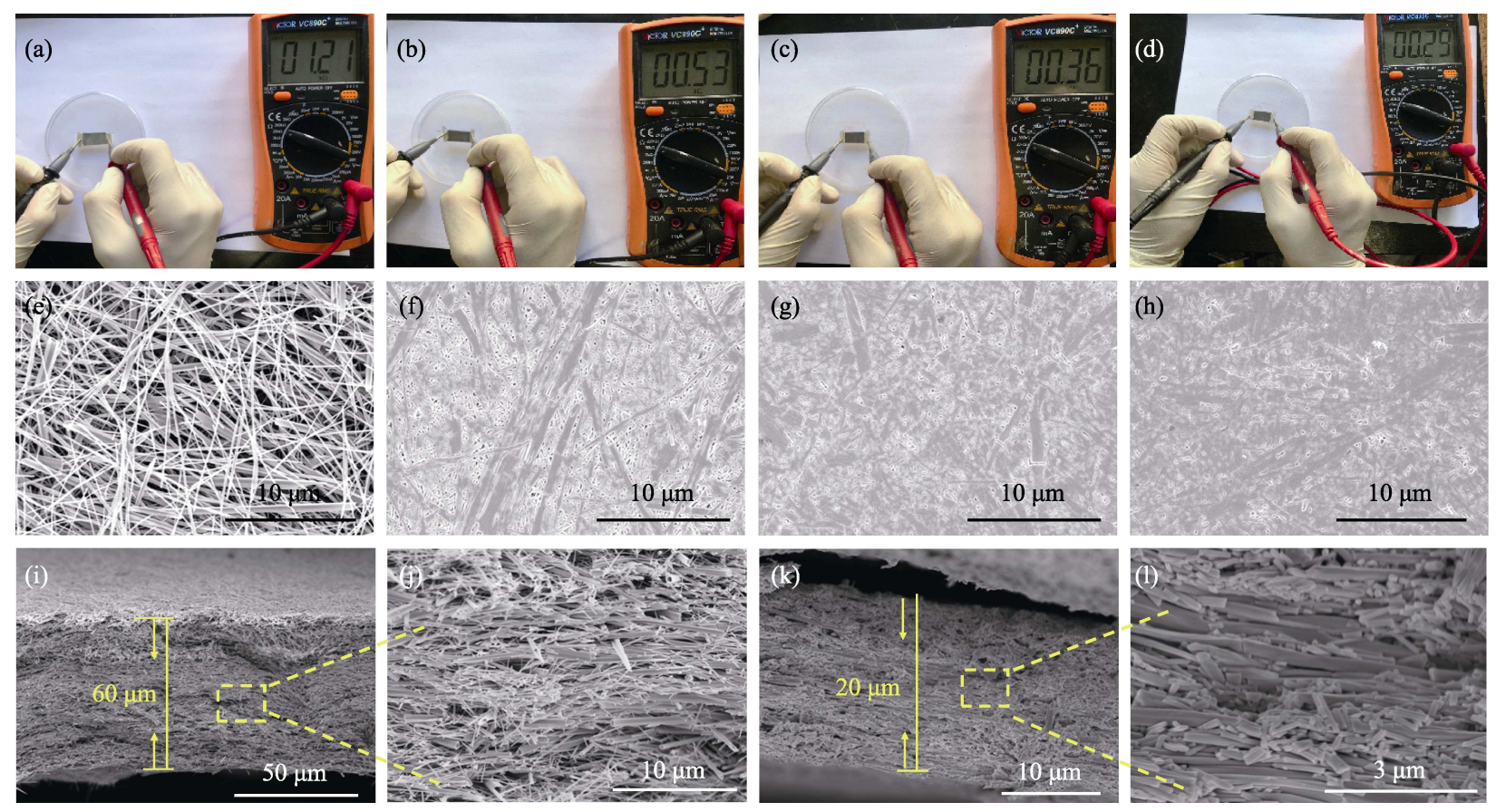

图 $6(\mathrm{a}, \mathrm{e})$ 未湿压处理以及在 $(\mathrm{b}, \mathrm{f}) 10$ 、 $(\mathrm{c}, \mathrm{g}) 20 、(\mathrm{~d}, \mathrm{~h}) 30 \mathrm{MPa}$ 湿压处理的 $\mathrm{TN} 160$ 薄膜 $(1 \mathrm{~cm} \times 2 \mathrm{~cm})$ 的 (a d) 电阻测试数码照片和 $(e \sim h)$ 薄膜表面 FESEM 照片; (i, j)未湿压处理和在 $(k, 1) 30 \mathrm{MPa}$ 湿压处理的

$\mathrm{TN} 160$ 薄膜的(i, k) 断面和(j, 1)局部放大 FESEM 照片

Fig. 6 (a-d) Digital photos of film $(1 \mathrm{~cm} \times 2 \mathrm{~cm})$ resistance test and $(\mathrm{e}-\mathrm{h})$ FESEM surface images of TN160 film (a, e) without and with wet press at (b, f)10, (c, g) 20, (d, h) $30 \mathrm{MPa}$; (i, k) Cross-sectional and (j, l) high-magnification cross-sectional FESEM images of TN160 films $(i, j)$ without and $(k, 1)$ with wet press at $30 \mathrm{MPa}$

湿压处理的薄膜的宏观电阻为 $0.36 \mathrm{k} \Omega$ (图 6(c)), 与 $10 \mathrm{MPa}$ 湿压处理的薄膜相比稍有降低, 薄膜微观致 密程度没有明显差异(图 6(g))。30 MPa 湿压处理的 薄膜如图 6(d, h) 所示, 薄膜的宏观电阻为 $0.29 \mathrm{k} \Omega$ 。 可见, 随着湿压处理压力的增大, 薄膜的致密程度 逐渐增强, 电阻随之呈现下降趋势。未湿压处理的 薄膜断面 FESEM 照片如图 6(i,j)所示, 其厚度约为 $60 \mu \mathrm{m}$ 。在 $30 \mathrm{MPa}$ 湿压处理的作用下薄膜变得致密, 厚度达到 $20 \mu \mathrm{m}$ (图 6(k, 1))。为了说明薄膜致密度的 具体变化, 对湿压处理后薄膜的体积密度进行测 试。通过测量薄膜的质量和薄膜的体积进行计算, 得到未处理、10、20、30 MPa 下处理的薄膜的体积 密度分别为 $1210 、 2918 、 3551 、 3940 \mathrm{~kg} \cdot \mathrm{m}^{-3}$, 可见
随着压力的增大, 薄膜的致密度逐渐增加。

对湿压处理后的 TN160 薄膜进行热电性能测 试, 如图 7 所示。随着湿压处理压力的增加, 薄膜的 电导率从 $26 \mathrm{~S} \cdot \mathrm{m}^{-1}$ 提升到 $476 \mathrm{~S} \cdot \mathrm{m}^{-1}$ (图 7(a)), 这也 佐证了湿压法能够增加碲纳米线薄膜的致密度并降 低电阻, 有助于热电性能的提升, 但与此同时薄膜 的塞贝克系数有一定程度的降低, 如图 7(b)所示。

图 7(c)测试了湿压处理对薄膜功率因子的影响, 当 压力达到 $30 \mathrm{MPa}$ 时, 薄膜的功率因子从 6.14 提升 到 $38 \mu \mathrm{W} \cdot \mathrm{m}^{-1} \cdot \mathrm{K}^{-2}$ 。

采用霍尔效应测试系统对 TN160薄膜的载流子 迁移率和载流子浓度进行测试, 从图 8 中可以看出, 湿压处理后薄膜的载流子迁移率和载流子浓度都有
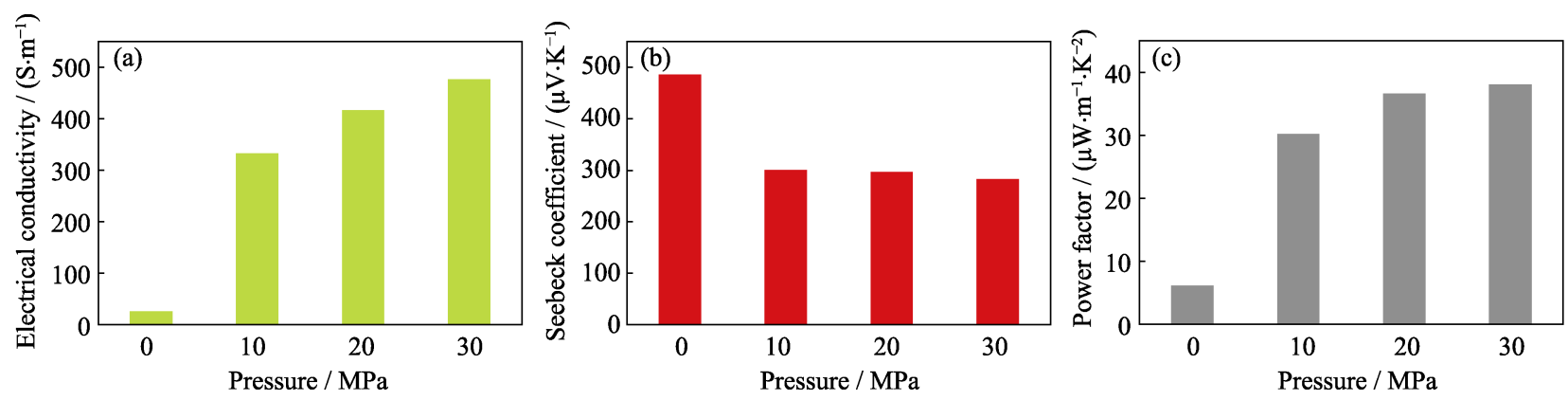

图 7 湿压处理前后 TN160 薄膜的(a)电导率、(b)塞贝克系数和(c)功率因子

Fig. 7 (a) Electrical conductivity, (b) Seebeck coefficient and (c) power factor of TN160 film before and after wet press 


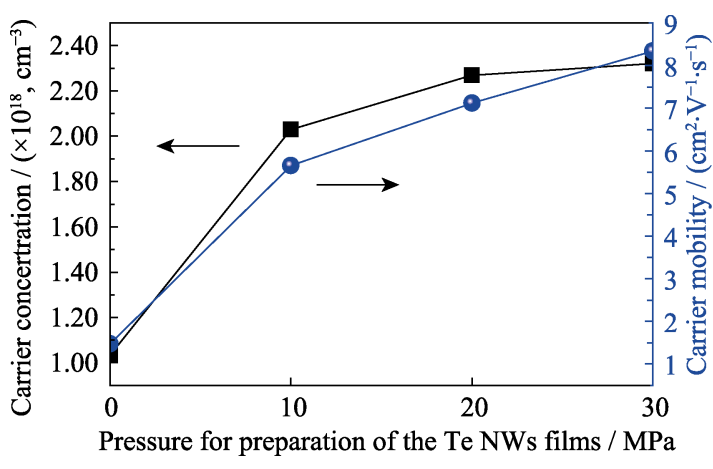

图 8 湿压处理后 $\mathrm{TN} 160$ 薄膜的载流子浓度和载流子迁移率 Fig. 8 Carrier concentration and carrier mobility of TN160 film with wet press at different pressures

\section{表 130 MPa 湿压处理的 TN160 薄膜柔性测试 \\ Table 1 Flexibilities of TN160 film with wet press at $30 \mathrm{MPa}$}

\begin{tabular}{cccc}
\hline $\begin{array}{c}\text { Curve } \\
\text { radius/ } \\
\mathrm{cm}\end{array}$ & $\begin{array}{c}\text { Electrical } \\
\text { conductivity/ } \\
\left(\mathrm{S} \cdot \mathrm{m}^{-1}\right)\end{array}$ & $\begin{array}{c}\text { Seebeck } \\
\text { coefficient/ } \\
\left(\mu \mathrm{V} \cdot \mathrm{K}^{-1}\right)\end{array}$ & $\begin{array}{c}\text { Power factor/ } \\
\left(\mu \mathrm{W} \cdot \mathrm{m}^{-1} \cdot \mathrm{K}^{-2}\right)\end{array}$ \\
\hline$\infty$ & 476 & 282.9 & 38 \\
2.0 & 472 & 282.0 & 37.5 \\
1.6 & 465 & 282.3 & 37.1 \\
1.2 & 458 & 282.0 & 36.4 \\
0.8 & 448 & 281.9 & 35.6 \\
\hline
\end{tabular}

所提高。随着湿压法压力的增大，薄膜内部致密度 逐渐提升, 导致纳米线之间的接触更加紧密, 有利 于载流子的传输, 所以薄膜的载流子迁移率逐渐提 高。因为薄膜的密度增加, 其载流子浓度也相应提 高 ${ }^{[23]}$ 。由于电导率与载流子迁移率和载流子浓度保 持正相关，因此薄膜的电导率也随之大幅度提高， 另一方面薄膜内部碲纳米线之间接触紧密，一定程 度上削弱了晶界对低能量载流子的过滤效应，导致 载流子浓度上升，降低了载流子的平均能量，所以 塞贝克系数有所降低。

对 $30 \mathrm{MPa}$ 湿压处理后的 TN160 薄膜进行柔性 测试(表 1), 分别测试了曲率半径为 2.0、1.6、1.2、 $0.8 \mathrm{~cm}$ 四种弯曲状态下薄膜的热电性能。如表 1 所 示, 当薄膜处于不同曲率半径的弯曲状态下时, 其 电导率、Seebeck 系数和功率因子均较为稳定。其 中, 薄膜的功率因子在弯折曲率半径达到 $0.8 \mathrm{~cm}$ 时, 相比于初始状态仅下降 $6 \%$, 说明本研究中的柔性 热电薄膜能够在弯折情况下保持良好的性能。

\section{3 结论}

1) 通过调控水热反应溶液的还原性强弱(抗坏 血酸添加与否), 制备了两种不同长径比的碲纳米
线; 弱还原性反应溶液(未添加抗坏血酸)中制备的 碲纳米线具有较高的长径比, 达到 200 。

2) 经水热法合成较大长径比的碲纳米线组装 的薄膜电导率可达 $26 \mathrm{~S} \cdot \mathrm{m}^{-1}$, 是较小长径比碲纳米 线薄膜的 8.4 倍。

3) 通过湿压法显著增大碲纳米线薄膜的微观 致密度，提高薄膜的载流子迁移率和载流子浓度， 大幅度改善薄膜的电导率，使薄膜的电导率提升了 18.3 倍。所得薄膜的电导率达到 $476 \mathrm{~S} \cdot \mathrm{m}^{-1}$, 塞贝克系 数达到 $282.9 \mu \mathrm{V} \cdot \mathrm{K}^{-1}$, 功率因子达到 $38 \mu \mathrm{W} \cdot \mathrm{m}^{-1} \cdot \mathrm{K}^{-2}$ 。

\section{参考文献:}

[1] CHEN L D, SHI X, LI Y L, et al. Congruent growth of $\mathrm{Cu}_{2} \mathrm{Se}$ thermoelectric thin films enabled by using high ablation fluence during pulsed laser deposition. J. Inorg. Mater, 2015, 30(10): $1115-1120$.

[2] FITRIANI, OVIK R, LONG B D, et al. A review on nanostructures of high-temperature thermoelectric materials for waste heat recovery. Renew. Sust. Energ. Rev., 2016, 64: 635-659.

[3] LEE J A, ALIEV A E, BYKVOA J S, et al. Woven-yarn thermoelectric textiles. Adv. Mater., 2016, 28(25): 5038-5044.

[4] HU G, MA J, ZOU J, et al. Dependence of the texture on the thermoelectric properties of C/C composites. J. Inorg. Mater, 2015, 4: 24-29.

[5] SIDDIQUE A R, MAHMUD S, HEYST B, et al. Review of the state of the science on wearable thermoelectric power generators (TEGs) and their existing challenges. Renew. Sust. Energ. Rev., 2017, 73: 730-744.

[6] GUO Y, DUN C, XU J, et al. Wearable thermoelectric devices based on Au-decorated two-dimensional $\mathrm{MoS}_{2}$. ACS Appl. Mater. Interfaces, 2018, 10(39): 33316-33321.

[7] HE M, LIN Y J, CHIU C M, et al. A flexible photo-thermoelectric nanogenerator based on $\mathrm{MoS}_{2} / \mathrm{PU}$ photothermal layer for infrared light harvesting. Nano Energy, 2018, 49: 588-595.

[8] DELAIZIR G, BERNARD G, MONNIER J, et al. A comparative study of Spark Plasma Sintering (SPS), Hot Isostatic Pressing (HIP) and microwaves sintering techniques on p-type $\mathrm{Bi}_{2} \mathrm{Te}_{3}$ thermoelectric properties. Mater. Res Bull., 2012, 47(8): 1954-1960.

[9] ZHAO C, ZHANG H, QI X, et al. Ultra-short pulse generation by a topological insulator based saturable absorber. Appl. Phys. Lett., 2012, 101(21): 251112.

[10] XU S, HONG M, SHI X L, et al. High-performance PEDOT:PSS flexible thermoelectric materials and their devices by triple post-treatments. Chem. Mater., 2019, 31(14): 5238-5244.

[11] BESSAIR B, MATHIEU M, SALLES V, et al. Synthesis of continuous conductive PEDOT:PSS nanofibers by electrospinning: a conformal coating for optoelectronics. ACS Appl. Mater. Interfaces, 2017, 9(1): 950-957.

[12] ABAD B, ALDA I, CHAO P, et al. Improved power factor of polyaniline nanocomposites with exfoliated graphene nanoplatelets (GNPs). J. Mater. Chem. A, 2013, 1(35): 10450-10457.

[13] LIU J, JIA Y, JIANG Q, et al. Highly conductive hydrogel polymer fibers toward promising wearable thermoelectric energy harvesting ACS Appl. Mater. Interfaces, 2018, 10(50): 44033-44040.

[14] WAN C, TIAN R, KONDOU M, et al. Ultrahigh thermoelectric power factor in flexible hybrid inorganic-organic superlattice. Nat. Commun., 2017, 8(1): 1024. 
[15] GAO J, LIU C, MIAO L, et al. Enhanced power factor in flexible reduced graphene oxide/nanowires hybrid films for thermoelectrics. RSC Advances, 2016, 6(38): 31580-31587.

[16] SONG H, CAI K. Preparation and properties of PEDOT:PSS/Te nanorod composite films for flexible thermoelectric power generator. Energy, 2017, 125: 519-525.

[17] SEE K C, FESER J P, CHEN C E, et al. Water-processable polymernanocrystal hybrids for thermoelectrics. Nano Lett., 2010, 10(11): 4664-4667.

[18] GAO J, LIU C, MIAO L, et al. Improved thermoelectric performance in flexible tellurium nanowires/reduced graphene oxide sandwich structure hybrid films. J. Electron. Mater, 2016, 46(5): 3049-3056.

[19] WU B, GUO Y, HOU C Y, et al. High-performance flexible thermoelectric devices based on all-inorganic hybrid films for harvesting low-grade heat. Adv. Funct. Mater., 2019, 29: 1900304.
[20] ZHONG B N, FEI G T, FU W B, et al. Solvothermal synthesis, stirring-assisted assembly and photoelectric performance of $\mathrm{Te}$ nanowires. Phys. Chem. Chem. Phys., 2016, 18(48): 32691-32696.

[21] ZHONG B N, FEI G T, FU W B, et al. Controlled solvothermal synthesis of single-crystal tellurium nanowires, nanotubes and trifold structures and their photoelectrical properties. CrystEngComm, 2017, 19(20): 2813-2820.

[22] LI Z, ZHENG S, ZHANG Y, et al. Controlled synthesis of tellurium nanowires and nanotubes via a facile, efficient, and relatively green solution phase method. J. Mater. Chem. A, 2013, 1(47): 15046-15052.

[23] GAO J, MIAO L, LIU C, et al. A novel glass-fiber-aided cold-press method for fabrication of n-type $\mathrm{Ag}_{2} \mathrm{Te}$ nanowires thermoelectric film on flexible copy-paper substrate. J. Mater. Chem. A, 2017, 5(47): 24740-24748. 